\title{
TEMPERATURE AND HUMIDITY EFFECTS OF DISPERSION MEDIUM ON EVOLUTION OF LIQUID-DROP AEROSOL PARAMETERS
}

\author{
Yuliya M. Stepkina ${ }^{1}$, Olga B. Kudryashova ${ }^{1}$, Boris V. Borisov ${ }^{2 a}$ \\ ${ }^{1}$ Institute for Problems of Chemical and Energetic Technologies, Siberian Branch of the Russian \\ Academy of Sciences, 659322 Biysk, Russia \\ ${ }^{2}$ Research Tomsk Polytechnic University, 634050 Tomsk, Russia
}

\begin{abstract}
A theoretical evaluation for the degree of temperature and humidity effects of dispersion medium on the evolution of liquid-drop aerosol parameters was represented in this work. A series of experiments to investigate parameters of aerosol media at different humidity and temperature of dispersion medium were conducted.
\end{abstract}

\section{INTRODUCTION}

The aerosol formation occurs upon mechanical comminution and atomization of solid bodies and liquids, crushing, attrition, explosions, burning, and pulverization. Whatever process produces the liquid-drop aerosol, its cloud undergoes further evolution: liquid droplets evaporate or expand during the vapor condensation; particles coalesce or break down, settle down.

Liquid-drop aerosols play a major role also in science, especially when particulates are applied to develop agricultural pesticides that are most often in the form of aerosols generated by atomization of dust-like or liquid preparations. Obscuring smoke and artificial fog are important for defense technology. The phenomenon of fog formation by condensation of oversaturated vapor on gaseous ions gave rise to one of the most important modern physical instruments, the Wilson chamber [1].

Mathematical modeling of thermal processes in the considered design avoids unnecessary costs in establishing the most effective modes of technology acquisition and development of high-silicate melt from the waste of energy and mining industry using the energy of low-temperature plasma. In this case, the total cross-sectional area, free to move in this direction of the flow varies from a minimum at the entrance to the flow of raw materials up to a maximum at the intersection of the axis of the cylindrical crucible and again to a minimum at the expiration of the finished melt (Fig. 2).

In accordance with a change in the geometry of the flow cross section and the flow rate varies. The minimum radial velocity is in the axis of the crucible, where the stream is most intense thermal effect, which favorably affects the final result, in terms of the completeness of the greatest melt. On the other hand achieve a melt temperature higher than necessary, leading to thermal decomposition of the raw material required to loss of useful properties. Preliminary analysis of the modes of fusion by means of mathematical modeling, taking into account the maximum design features allowing for an early design stage to choose the most rational modes of loading of raw materials, melt and discharge parameters of the plasma column.

${ }^{\mathrm{a} C}$ Corresponding author: bvborisov@tpu.ru 


\section{THEORETICAL}

Evaporation processes may become essential for liquid-drop aerosols. Evaporation processes have the largest influence on particles less than $10 \mu \mathrm{m}$ in size; so, these processes are necessary to take into account when considering an ensemble of such particles. Such influence is governed by developed heat exchange with the environment and by energy entrainment from a relatively large surface of droplets.

The evaporation dynamics of small droplets is defined by the Maxwell equation [2]:

$$
I=\frac{d m}{d t}=\frac{4 \pi r D_{f} M\left(p_{d r o p}-p_{p l}\right)}{R T},
$$

where $m=\frac{4}{3} \pi r^{3} \rho_{\kappa}$ - the droplet weight; $D_{f}-$ the diffusion coefficient; $M-$ the molecular weight of the liquid; $T$ - the absolute temperature; $r$ - the particle radius; $\rho_{k}-$ the droplet density, $R-$ the

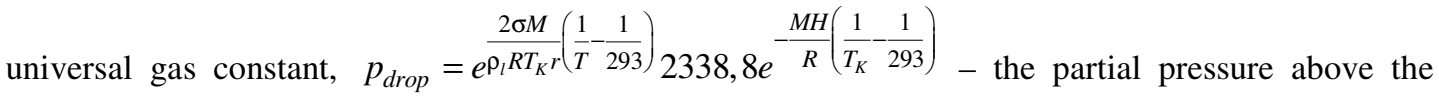
droplet; $\sigma-$ the surface tension coefficient of the droplet matter; $T_{\kappa}-$ the droplet temperature; $p_{p l}=v 2338,8 e^{-\frac{M H}{R}\left(\frac{1}{T_{\infty}}-\frac{1}{293}\right)}-$ the partial pressure above the plain surface; $v$ - humidity; $T_{\infty}-$ the air temperature.

From (1) can be derived a differential equation for the rate of the droplet weight change caused by evaporation [3]:

$$
\frac{d r}{d t}=-\frac{D_{f} M r}{2 \rho_{l} R T_{\infty}} 2338,8\left(e^{\frac{2 \sigma M}{\rho_{l} R T_{K} r}} e^{-\frac{M H}{R}\left(\frac{1}{T_{K}}-\frac{1}{293}\right)}-v e^{-\frac{M H}{R}\left(\frac{1}{T_{\infty}}-\frac{1}{293}\right)}\right)
$$

The evaporation time is calculated by expressing $t$ from equation (2):

$$
t=\frac{r^{2}}{\frac{6 D_{f} M}{\rho_{\kappa} R T_{\infty}} e^{-\frac{M H}{R}\left(\frac{1}{T_{K}}-\frac{1}{293}\right)} 2338,8\left(e^{\frac{2 \sigma M}{\rho_{K} R T_{K} \cdot r}}-v\right)}
$$

A theoretical research is then pursued for the evaporation time of the liquid-drop aerosol mainly composed of distilled water, with a particle size of 1 to $5 \mu \mathrm{m}$. In the calculation of the evaporation time of the aqueous aerosol by formula (3), the simplification $T_{\kappa}=T_{\infty}$ is taken as well as the basic parameters for distilled water: $D_{f}=2,2 \cdot 10^{-5} \mathrm{~m}^{2} / \mathrm{s} ; M=0,018 \mathrm{~kg} / \mathrm{mol} ; \mathrm{D}=1-15 \mu \mathrm{m} ; \rho_{l}=998 \mathrm{~kg} / \mathrm{m}^{3}$; $R=8,31 \mathrm{~m}^{2} \cdot \mathrm{kg} \cdot \mathrm{s}^{2} \cdot \mathrm{K}^{-1} \cdot \mathrm{mol}^{-1} ; T_{\kappa}=T_{\infty}=0-50^{\circ} \mathrm{C} ; \sigma=0,0728 \mathrm{~N} / \mathrm{m} ; \mathrm{H}=2257000 \mathrm{~J} / \mathrm{kg}$ [4].

As an example of the calculation, we plot the evaporation time of the aerosol particles having a size of 1 to $15 \mu \mathrm{m}$ against the dispersion medium humidity at a high humidity of 90\% (Fig. 1) [4].

Figure 2 illustrates a plot of the evaporation time of the aerosol particles $1-15 \mu \mathrm{m}$ in diameter versus the dispersion medium temperature at $T_{\kappa}=30^{\circ} \mathrm{C}$. 


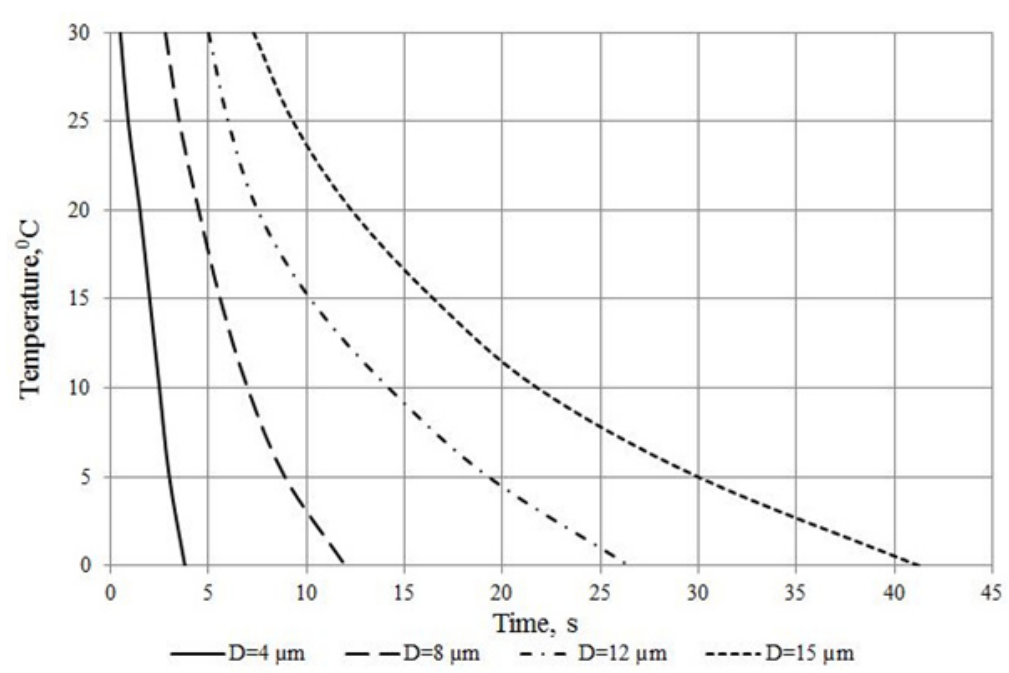

Figure 1. The aqueous aerosol evaporation time at the dispersion medium humidity equal to $90 \%$.

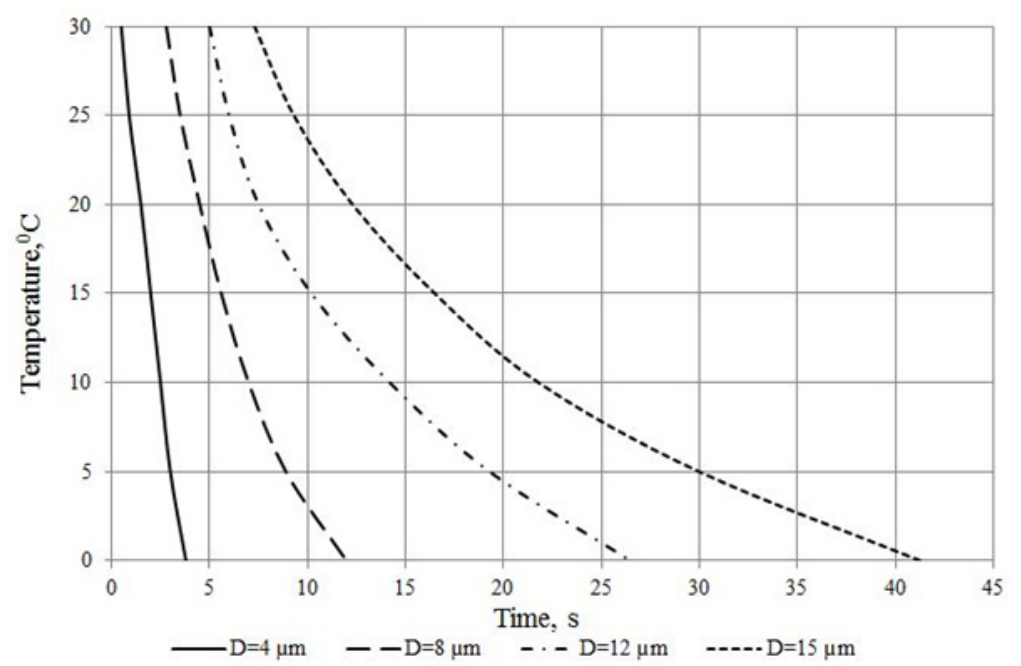

Figure 2. The aqueous aerosol evaporation time at the dispersion medium temperature equal to $30^{\circ} \mathrm{C}$.

\section{Experimental}

The experimental research of influence of temperature and humidity effects of the dispersion medium on the evolution of liquid-drop aerosol parameters was conducted in a climatic heat-cold-moisture chamber (HCMC-10, KTXB-10 in Russian). A paint-spray gun (KRATON R-200 LVLP-02S) was used for generation a liquid-drop aerosol. The evolution of the liquid-drop aerosol parameters in the experimental climatic chamber was studied using a LID-2M measuring complex since the complex is designed for measuring dispersiveness and concentration of aerosols from the moment of the aerosol genesis until complete disappearance. The operation principle of the LID-2M setup relies on recording the overall intensity of the laser radiation scattered on the dispersed particles [5].

The weight of the atomized water in the experiments was $20 \mathrm{~g}$ and the atomization time was $1 \mathrm{~s}$.

The experiments with the aid of the LID-2M software resulted in parameters $a$ and $b$ for the gamma distribution at various moments of time [5]. For illustrative purposes, the distribution function 
should be presented for the time moments $t=19.51 \mathrm{~s}, t=30.03 \mathrm{c}, t=35.65 \mathrm{~s}$, and $t=135 \mathrm{~s}$ at a dispersion medium humidity of $70 \%$ and temperature of $30^{\circ} \mathrm{C}$ (Fig. 3).

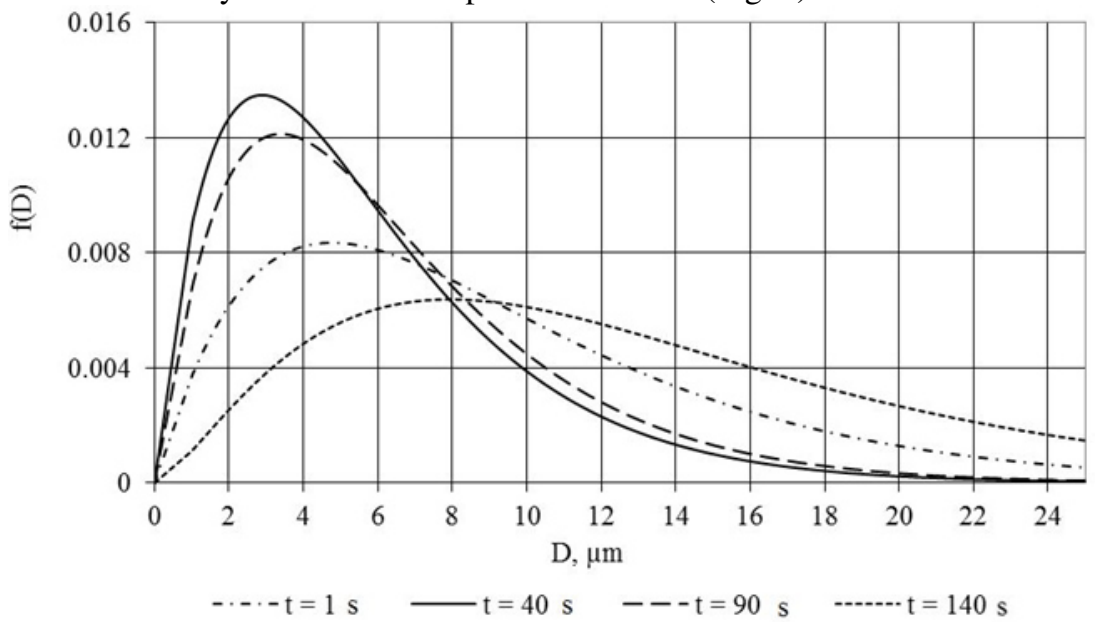

Figure 3. Distilled water particle size distribution function at $70 \%$ humidity and $30 \mathrm{o} C$ of the dispersion medium.

From the analysis of Figure 3, it can be deduced that the maximum of the distribution function shifts with time towards smaller particle diameters due to evaporation.

The particle mass concentration $\mathrm{Cm}$ is important characteristic for description of the aerosol evolution process, which can be identified using the LID-2M setup. The method to determine the particle concentration is based on measuring spectral transmittance of the two-phase flow for some fixed wavelength $\lambda$ of the prove radiation [5].

Figure 4 shows a graph of the variation of the particle mass concentration $\mathrm{Cm}$ with time at a dispersion medium temperature of $30^{\circ} \mathrm{C}$ and humidity of $60 \%$.

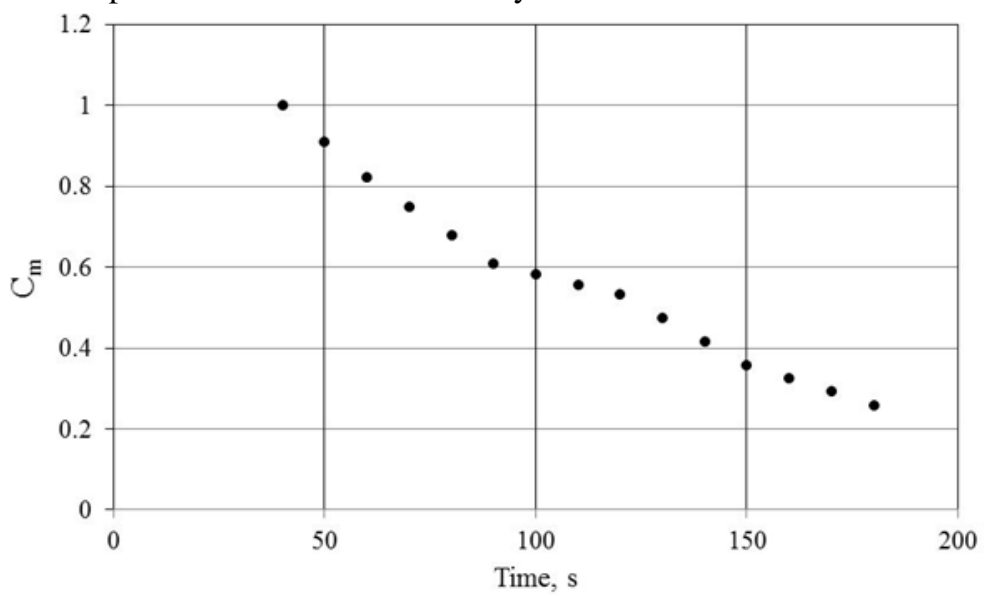

Figure 4. The mass concentration of aqueous aerosol particles versus time at $60 \%$ humidity and $20^{\circ} \mathrm{C}$ of the dispersion medium.

\section{Conclusion}

The theoretical evaluation of temperature and humidity effects of the dispersion medium on the evolution of liquid-drop aerosol parameters has been performed that enables predicting the aqueous aerosol evaporation time. It has consequently been found that the rise in humidity significantly 
increases the particle evaporation time, as does the decrease in temperature; the evaporation time is much less influenced by the particle size; so, the determinative factors of the evolution process of the liquid-drop aerosol parameters are particularly temperature and humidity of the dispersion medium. The experimental values obtained for the particle size distribution function and mass concentration by means of the laser measuring setup allow specific values of time to be determined when the most of distilled water particles have evaporated from the working volume, in order to solve practical problems.

\section{References}

1. V.A. Arkhipov, Aerosol systems and their effect on vital activity, Tomsk: GTPU, 2007, p. 136.

2. V.I. Roldugin, Physicochemistry of surface, Publishing House "Intellekt", 2008, Pp. 365.

3. R.I. Nigmatulin, Dynamics of multiphase media. Part 1, M: Nauka, 1987, Pp. 345.

4. P.S. Prokhorov, V.N. Yashin, On the causes of noncoalescence of water droplets upon collisions, Colloid Journal, 1948, pp. 122-124.

5. I.R. Akhmadeev, Method and fast-acting laser setup to study the genesis of technogenic aerosols from beam scattering in a controllable volume, Candidate of Engineering Science thesis, Altai State Technological University, Barnaul, 2008, p. 98. 